\title{
Cryptocurrency User Attitudes Towards the Environmental Impact of Proof-of-Work
}

\author{
Moritz Platt 1,2,*iD, Francesco Pierangeli 1,3,4 \\ 1 Centre for Blockchain Technologies, University College London \\ 2 Department of Informatics, King's College London \\ 3 Department of Political Economy, King's College London \\ 4 Department of Economics, National University of Singapore \\ * Correspondence: moritz.platt@kcl.ac.uk; Tel.: +44 2078365454
}

\begin{abstract}
The consumption of electrical energy is a requisite for 'proof-of-work', a class of consensus protocols for decentralised systems. 'Ethereum' and 'Bitcoin', along with various other blockchains, use implementations of such a consensus protocol. Among experts, the vast energy demand associated with the rising popularity of cryptocurrencies and the potential impact on climate change have been discussed extensively. It is, however, unclear what attitudes the users of cryptocurrencies themselves have towards the consequences of its growing energy demand. The proposed study aims to answer this question through survey research, using 'Bitcoin' as an archetype of a proof-of-work cryptocurrency. Conducting the study will reveal whether cryptocurrency users themselves consider their energy needs to be problematic, and which stakeholders they hold accountable to reduce consumption. The outcome can provide a theoretical grounding in social science for the ongoing implementation of alternative consensus models, for example in the context of the 'Eth2' upgrade of the 'Ethereum' blockchain.
\end{abstract}

Keywords: Cryptocurrency, Bitcoin, Proof-of-Work, Mining

\section{Introduction}

The concept of a digital medium of exchange that doesn't rely on trusted third parties like financial institutions was discussed as early as the 1990s [1]. 2008 saw the first notable concrete implementation of this concept in the form of 'Bitcoin', a decentralised electronic cash system [2]. Bitcoin uses owner-signed digital coins, a method that had previously been proposed to control ownership of electronic cash using trusted third parties [3]. In absence of those, this method, however, creates a risk of 'double-spending', a type of attack in which malicious participants can overspend their funds. To mitigate such an attack, the concept of a public history of transactions to which only those can append data who expend computational resources is introduced by Nakamoto [2]. This method is known as proof-of-work and, in the case of Bitcoin, uses a mechanism with scalable complexity [4]. The extent to which computational resources have to be expended is dynamically adapted by the protocol to target a constant number of transactions per hour [2]. This makes the protocol anti-efficient from an energy consumption perspective, as an increase in coin market capitalisation is correlated with an increase in power consumption [5]. Since its inception, Bitcoin has experienced a dramatic rise in popularity. While initially seen as a niche technology, interest in which was driven by computer programming enthusiasts and illegal activity [6], Bitcoin has recently reached a market capitalisation of US $\$ 1$ trillion providing evidence of its recognition beyond expert circles [7].

As described earlier, the nature of Bitcoin's underlying proof-of-work consensus mechanism means that an increase in price influences the need for expending energy [8]. The downsides of this need for energy consumption have been recognised early and alternative mechanisms, foremost 'proof-of-stake' $[9,10]$ have been proposed. Proofof-stake is now embraced by prominent public blockchains such as the decentralised application platform 'Ethereum' that is in the process of introducing a finality system 
based on this consensus mechanism [11]. Still, proof-of-work based systems, most importantly, Bitcoin, remain a mainstay. While the energy consumption of Bitcoin cannot be measured due to its decentralised nature, estimates can be made based on several observable factors. The Cambridge Centre for Alternative Finance [8] provides a model based on six dynamic factors and three static factors that provides an estimated annualised electricity consumption of the Bitcoin network of $129.24 \mathrm{TWh}$, with a theoretical lower bound of 40.45 TWh and a theoretical upper bound of 444.55 TWh when extrapolating based on the factors as determined on 18 March 2021. While these figures-as well as projections based on them that are out of the scope of this proposal-have caused scientists to express concern about the contribution of Bitcoin to global $\mathrm{CO}_{2}$ emissions [12], little is know about the attitude of Bitcoin users towards this problem.

\section{Study Goals and Objectives}

The proposed study uses Bitcoin as an archetype of a proof-of-work cryptocurrency due to it being the most highly capitalised cryptocurrency [7] and one that highly popular amongst internet users [13]. Nonetheless, the results generated should be generic enough for the to be applicable to other decentralised protocols, specifically, those that are actively undergoing changes in their consensus mechanisms like the Ethereum blockchain in the context of the 'Eth2' update.

The primary objective of the proposed study is to determine what attitude Bitcoin users have towards the environmental impact of this cryptocurrency. Specifically, the following questions can be derived from this: Do users understand that running proofof-work entails consuming amounts of electricity equivalent to that of highly developed nations? Do users make a connection between the electricity consumption of proof-ofwork and global warming? And, if they do make such a connection: Do they see an acute need for action to limit climate impact? And if so, by whom?

\section{Study design}

The research question will be approached via a survey with closed-ended questions. The target population are Bitcoin users, defined as those that have participated in one or more Bitcoin transactions over the past 5 years. This population will be targeted by a questionnaire that will be presented to a broader population using a market research provider. The intended sequence of questions is outlined in figure 1. Initially, the experience of participants is determined by asking them for the number of transactions they have participated in. Should the participant be unaware of the concept of a Bitcoin transaction, or, should they have never participated in one, the questionnaire will be terminated for them. Next, the questionnaire will establish how realistic the participant's estimation of Bitcoin's energy consumption is. Based on the source described earlier [8], the correct answer to the question is 'Roughly the equivalent of what the entirety of Sweden consumes'. Alternatives to this are deliberately exaggerated to determine whether the participant severely over or underestimates the energy requirements. Next, it is established whether the participant believes in a link between Bitcoin's energy consumption and global warming. If they do, they are asked whether, in their view, this leads to the need for measures being taken. Depending on their choice they are then presented with reasons for not taking measures or actors that they see as having a responsibility to act. 


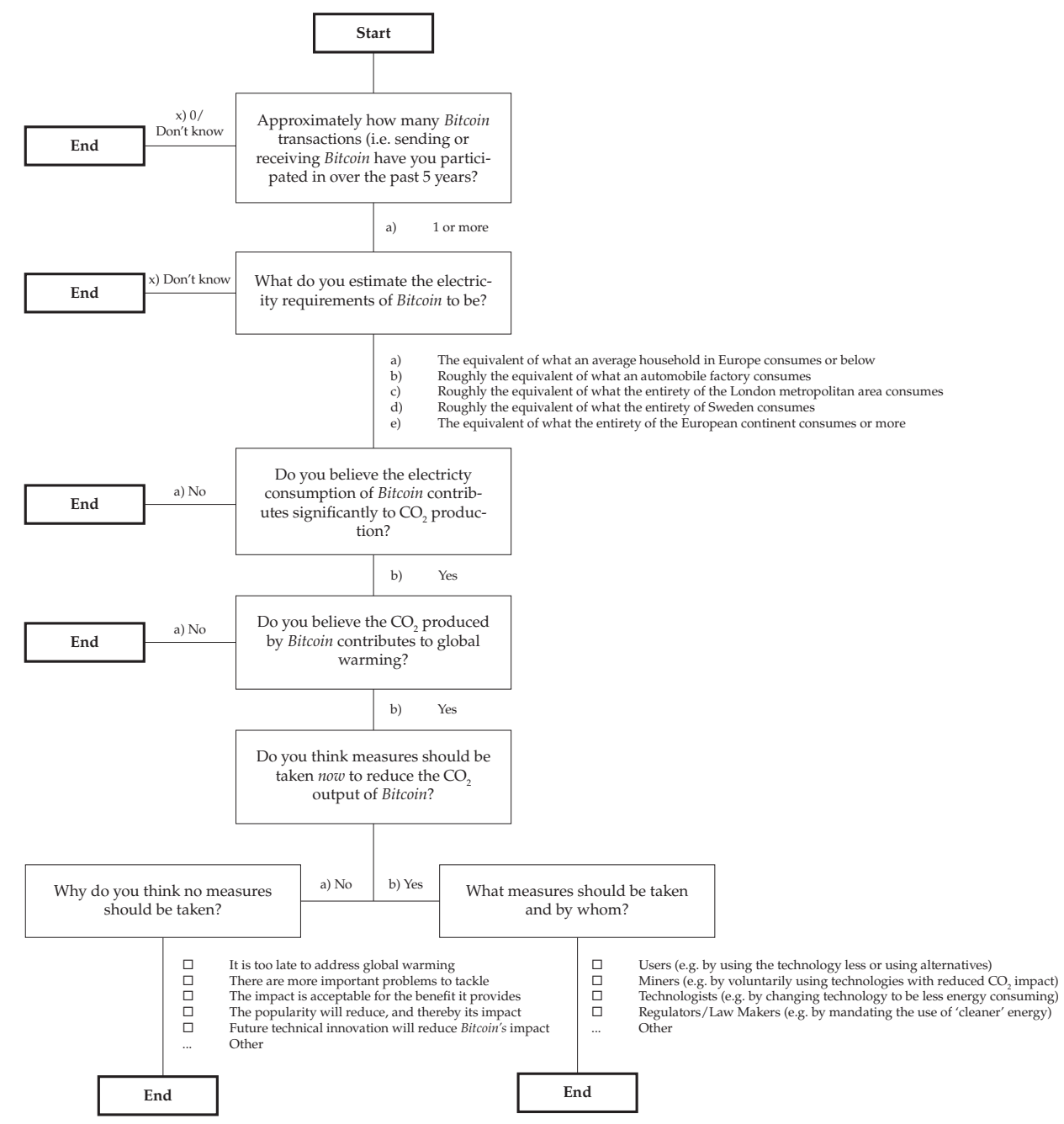

Figure 1. A flow chart describing the intended questionnaire design.

\section{Impact}

Answering these research questions can provide an important impetus for technologists, such as the developers of novel blockchain protocols and those undertaking changes to existing ones. Furthermore, the results can validate the work around the development of alternative consensus mechanisms, and legitimise further research in this area. The findings can also be used by policymakers and regulators to develop strategies for engaging with cryptocurrencies.

\section{References}

1. Dai, W. b-money. http://www.weidai.com/bmoney.txt, accessed on 17 November 2020.

2. Nakamoto, S. Bitcoin: A peer-to-peer electronic cash system. https:// bitcoin.org/bitcoin.pdf, accessed on 10 October 2020.

3. Chaum, D.; Fiat, A.; Naor, M. Untraceable Electronic Cash. Proceedings of the 1988 Conference on the Theory and Application of Cryptography; Goldwasser, S., Ed.; Springer New York: Santa Barbara, CA, USA, 1990; CRYPTO, pp. $319-327$.

4. Back, A. A partial hash collision based postage scheme. http://www.hashcash.org/papers/announce.txt, accessed on 18 March 2021.

5. Sedlmeir, J.; Buhl, H.U.; Fridgen, G.; Keller, R. The Energy Consumption of Blockchain Technology: Beyond Myth. Business $\mathcal{E}$ Information Systems Engineering 2020, 62, 599-608.

6. Yelowitz, A.; Wilson, M. Characteristics of Bitcoin users: an analysis of Google search data. Applied Economics Letters 2015, 22, 1030-1036.

7. Lam, E. Bitcoin Hits $\$ 1$ Trillion Value as Crypto Leads Other Assets. https://www.bloombergquint.com/crypto/bitcoin-nears-1trillion-value-as-crypto-jump-tops-other-assets, accessed on 18 March 2021.

8. Cambridge Centre for Alternative Finance. Cambridge Bitcoin Electricity Consumption Index. https://cbeci.org, accessed on 18 March 2021. 
9. QuantumMechanic. Proof of stake instead of proof of work. https:// bitcointalk.org/index.php?topic=27787.0, accessed on 22 May 2020.

10. King, S.; Nadal, S. PPCoin: Peer-to-Peer Crypto-Currency with Proof-of-Stake. https://decred.org/research/king2012.pdf, accessed on 19 January 2020.

11. Buterin, V.; Griffith, V. Casper the Friendly Finality Gadget. https://arxiv.org/abs/1710.09437, accessed on 11 August 2020. arXiv:1710.09437.

12. Mora, C.; Rollins, R.L.; Taladay, K.; Kantar, M.B.; Chock, M.K.; Shimada, M.; Franklin, E.C. Bitcoin emissions alone could push global warming above $2^{\circ} \mathrm{C}$. Nature Climate Change 2018, 8, 931-933. doi:10.1038/s41558-018-0321-8.

13. Park, S.; Park, H.W. Diffusion of cryptocurrencies: web traffic and social network attributes as indicators of cryptocurrency performance. Quality \& Quantity 2019, 54, 297-314. doi:10.1007/s11135-019-00840-6. 